\title{
Adenoviral-mediated GDNF protects bone marrow mesenchymal stem cells against apoptosis induced by hydrogen peroxide
}

\author{
Xiaoqing Gao ${ }^{\text {a }}$, Jie Du ${ }^{\mathrm{a}}$, Guangbi Fan ${ }^{\mathrm{a}}$, Chaoxian Yang ${ }^{\mathrm{a}}$, Li Deng ${ }^{\mathrm{a}}$ and Qionglan Yuan ${ }^{\mathrm{b},}$ \\ ${ }^{a}$ Department of Anatomy and Neurobiology, Luzhou Medical College, Sichuan Luzhou 646000, China \\ ${ }^{b}$ Department of Anatomy and Neurobiology, Tongji University School of Medicine, Shanghai 200092, \\ China
}

\begin{abstract}
In this study, the effects of adenoviral-mediated glial cell line-derived neurotrophic factor (Ad-GDNF) on apoptosis of cultured bone marrow mesenchymal stem cells (BMSCs) induced by hydrogen peroxide $\left(\mathrm{H}_{2} \mathrm{O}_{2}\right)$ were investigated. After BMSCs infected with Ad-GDNF were treated with $500 \mu \mathrm{M} \mathrm{H}_{2} \mathrm{O}_{2}$ at $37^{\circ} \mathrm{C}$ for $1 \mathrm{~h}$, lactate dehydrogenase (LDH), MTT and TUNEL methods were used to detect cell viability and apoptosis. In addition, the levels of GDNF in the supernatants in BMSCs cultures were detected by ELISA, pro-inflammatory cytokines IL- 6 and TNF- $\alpha$ in the supernatants and apoptosis-related protein $\mathrm{Bax}$ and $\mathrm{Bcl}-2$ in cell pellets were investigated. The results showed that $\mathrm{H}_{2} \mathrm{O}_{2}$ treatment significantly induced apoptosis of BMSCs and decreased the viability of BMSCs. However, Ad-GDNF significantly reversed the effects of $\mathrm{H}_{2} \mathrm{O}_{2}$ on BMSCs. Furthermore, Ad-GDNF significantly decreased the levels of IL- 6 and TNF- $\alpha$ and increased Bcl-2/Bax ratio in BMSCs treated by $\mathrm{H}_{2} \mathrm{O}_{2}$. In conclusion, Ad-GDNF inhibits apoptosis of BMSCs induced by $\mathrm{H}_{2} \mathrm{O}_{2}$, and the mechanisms may be related to down-regulating the expression of IL- 6 and TNF- $\alpha$ and up-regulating the ratio of Bcl-2/Bax in BMSCs.
\end{abstract}

Keywords: Glial cell line-derived neurotrophic factor, bone marrow mesenchymal stem cells, hydrogen peroxide, apoptosis

\section{Introduction}

Transplantation of BMSCs has been demonstrated a great promising therapeutic potential for various central nervous system (CNS) diseases. But adverse microenvironments in injured CNS such as oxidative stress, believed to be central events in the pathological process [1], have negative effects on the survival of transplanted BMSCs. GDNF, a potent neurotrophic factor, can affect neuronal development, survival, outgrowth and have neuroprotective effects against various neuronal insults [2-5]. Indeed, it has previously been proved that BMSCs infected with Ad-GDNF (GDNF/BMSCs) were co-cultured with neurons subjected to hypoxia, the neuronal apoptosis were decreased compared

\footnotetext{
${ }^{*}$ Corresponding author: Qionglan Yuan, Department of Anatomy and Neurobiology, Tongji University School of Medicine, Shanghai 200092, China. Tel.: 021-65985626; Fax: 021-69584636; E-mail: yqiongl@263.net.
} 
with the neurons subjected to hypoxia alone; and the transplantation of GDNF/BMSCs also ameliorated significantly functional deficits and decreased apoptotic cells compared with transplantation of BMSCs after cerebral hemorrhage [6]. However, the underlying mechanism how GDNF relieves functional deficits and decreases cell apoptosis remains largely unknown. This study was designed to discuss the protective effect and possible mechanisms of Ad-GDNF against $\mathrm{H}_{2} \mathrm{O}_{2}$-induced apoptosis of BMSCs, which will conduce to further understanding of the therapeutic use of GDNF/BMSCs in transplantation strategies for central nervous system diseases, especially involved oxidative stress.

\section{Materials and methods}

\subsection{Reagents and apparatuses}

DMEM medium, 3-(4,5-Dimethylthiazol-2-yl)-2,5-diphenyltetrazolium bromide (MTT), 4', 6-diamidino-2-phenyl-indole (DAPI) (Sigma, USA); fetal bovine serum (FBS, Hyclone, USA); enzyme-linked immunosorbent assay (ELISA) Kit (Beijing Yonghui Biological Technology Co., Ltd., Beijing, China); The terminal deoxynucleotidyl transferase (TdT)-mediated dUTP-biotin nick end labeling (TUNEL) in situ cell death detection kit-POD (Roche Diagnostics Gmbh, Mannheim, Germany), Lactate dehydrogenase assay (LDH) Kit (Nanjing Jiancheng Bioengineering Institute, Nanjing, China); microplate reader (BioTeK, ELX800, Vermont, American), Olympus Fluorescence microscope (Japan).

\subsection{Culture and infection of BMSCS}

GDNF recombinant adenovirus vector (pAdEasy-1-pAdTrack CMV-GDNF, Adenoviral-mediated GDNF, Ad-GDNF) was prepared as described previously, and the viral titres were $1 \times 10^{9} \mathrm{PFU} / \mathrm{mL}$ and the infection rate was $100 \%$ [6,7]. BMSCs of C57BL/6 mouse (MUBMX-01001) were purchased from Cyagen Biosciences Inc, Guangzhou, China. The infection of BMSCs was performed as previously described [8]. Briefly, BMSCs were plated in DMEM medium containing $10 \%$ FBS at a density of $1 \times 10^{5}$ cells $/ \mathrm{mL}$ in $50 \mathrm{~mL}$ flask in a humidified environment with $5 \% \mathrm{CO}_{2}$ at $37^{\circ} \mathrm{C}$. When the BMSCs were at $80 \%$ confluence, $3 \mathrm{~mL}$ DMEM and $120 \mu \mathrm{L}$ GDNF virus solutions were added to each flask and were further incubated for $2 \mathrm{~d}$ to obtain GDNF/BMSCs.

\subsection{MTT assay}

Cell survival was determined using the MTT assay. BMSCs and GDNF/BMSCs were respectively plated at a density of $5 \times 10^{3}$ cells per well in $200 \mu \mathrm{L}$ DMEM in 96-well plates and incubated for $24 \mathrm{~h}$. Then cells were explored to $500 \mu \mathrm{M}$ hydrogen peroxide $\left(\mathrm{H}_{2} \mathrm{O}_{2}\right)$ for $1 \mathrm{~h}$. After washing with PBS, cells were incubated with $150 \mu \mathrm{L}$ medium and $20 \mu \mathrm{L} 0.5 \%$ MTT at $37^{\circ} \mathrm{C}$ for 4 hours. The media were removed, and $150 \mu \mathrm{L}$ dimethyl sulphoxide were added to each well. The absorbances at $490 \mathrm{~nm}$ wavelengths were read using a microplate reader. BMSCs without any treatment were subjected to MTT assay served as a control. 


\section{4. $L D H$ release assay}

The amounts of LDH in the medium reflect the loss of membrane integrity, apoptosis, or necrosis. $\mathrm{H}_{2} \mathrm{O}_{2}$-induced cytotoxicity was evaluated by measuring the quantities of LDH released into the medium from $\mathrm{H}_{2} \mathrm{O}_{2}$-injured BMSCs. Briefly, BMSCs and GDNF/BMSCs were explored to $500 \mu \mathrm{M}$ $\mathrm{H}_{2} \mathrm{O}_{2}$ for $1 \mathrm{~h}$, and the supernatants were used for LDH activity analysis following the commercially available LDH assay Kit protocol. The absorbances of the reaction mixture were measured at $450 \mathrm{~nm}$ wavelengths on the microplate reader.

\subsection{DAPI staining and TUNEL assay}

The apoptosis induced by $500 \mu \mathrm{M} \mathrm{H}_{2} \mathrm{O}_{2}$ was determined by morphological observations done with DAPI staining and TUNEL assay. BMSCs and GDNF/BMSCs were treated with $500 \mu \mathrm{M} \mathrm{H}_{2} \mathrm{O}_{2}$ for $1 \mathrm{~h}$ and then fixed in $4 \%$ paraformaldehyde for $30 \mathrm{~min}$ at room temperature. The cells were dyed with DAPI staining Kit for $10 \mathrm{~min}$, and washed with distilled water twice, then observed using a fluorescence microscope. TUNEL assay was determined by TUNEL in situ cell death detection kit-POD according to the manufacturer's protocol. Endogenous peroxidase activity was quenched with $3 \% \mathrm{H}_{2} \mathrm{O}_{2}$ in methanol for $10 \mathrm{~min}$ and permeabilization was performed with $0.1 \%$ Triton $\mathrm{X}-100$ in $0.1 \%$ sodium citrate for $10 \mathrm{~min}$. Then TUNEL reagent was added to each well and was incubated for one hour at $37^{\circ} \mathrm{C}$ in the dark. After converter-POD was added on the cells for the reaction at $37^{\circ} \mathrm{C}$ for $30 \mathrm{~min}$, the samples were spotted with $\mathrm{DAB}$ and hematoxylin at the room temperature.

\subsection{ELISA assay}

ELISA assay was used to detect: neurotrophic factors, GDNF; pro-inflammatory cytokines, IL-6 and TNF- $\alpha$; and apoptosis-related protein, Bax and Bcl-2. Briefly, BMSCs were infected with Ad-GDNF for $2 \mathrm{~d}$ to get the GDNF/BMSCs, and then were passaged at $1: 2$ using $0.25 \%$ trypsin solution. The supernatants of uninfected BMSCs and the end of passage 1, 2, and 3 of GDNF/BMSCs were collected for GDNF analysis. After BMSCs and GDNF/BMSCs had been treated with $500 \mu \mathrm{M}$ $\mathrm{H}_{2} \mathrm{O}_{2}$ for $1 \mathrm{~h}$ and then the cultures were centrifuged. The supernatants were collected for IL-6 and TNF- $\alpha$ analysis, and cell pellets were collected and repeated freezing and melting three times for Bax and Bcl-2 analysis. ELISA assay was performed following the manufacturer's protocol. The absorbances at $450 \mathrm{~nm}$ wavelength were determined using a microplate reader. According to the absorbances of the standard sample and the corresponding concentration value, a standard curve was drawn and the concentrations of GDNF, IL- 6 , TNF- $\alpha$, Bax and Bcl-2 were calculated.

\subsection{Statistical analysis}

The data were expressed as mean $\pm \mathrm{SD}$. The data were from five independent experiments. A statistical comparison was performed using one-way ANOVA. $\mathrm{P}$ value $<0.05$ was considered statistically significant. 

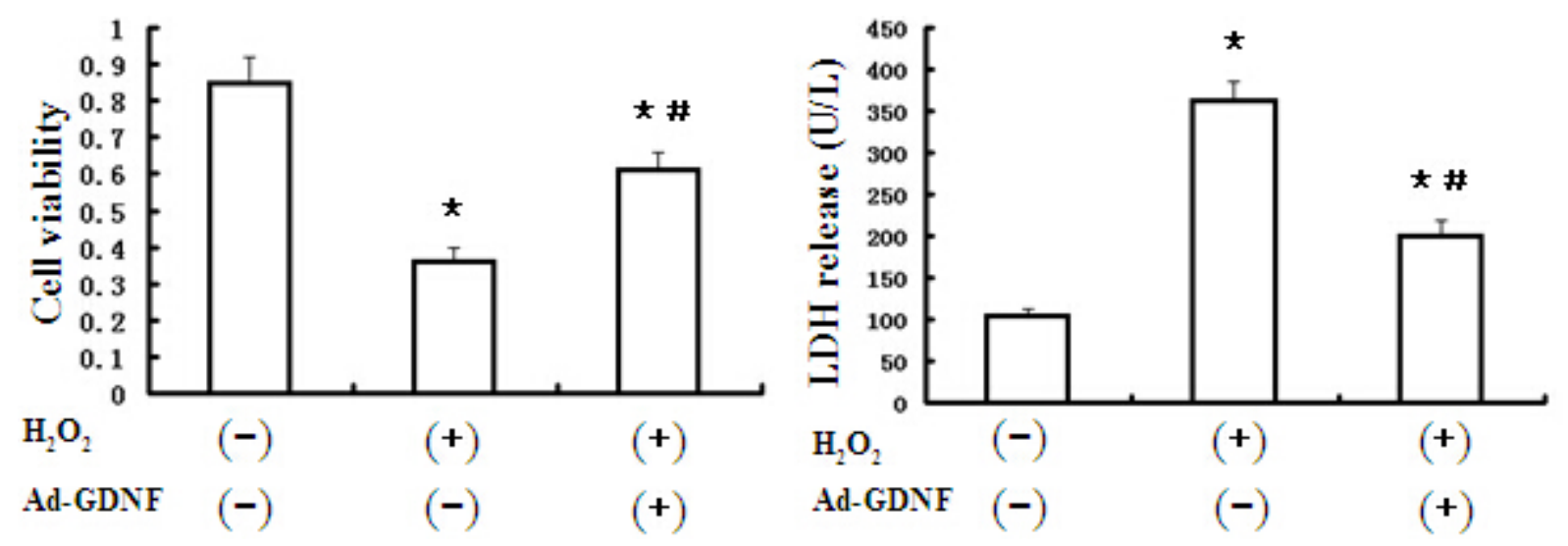

Fig. 1. Promoting effect of Ad-GDNF on the viability and LDH release of BMSCs under $\mathrm{H}_{2} \mathrm{O}_{2}$-induced oxidative stress. Data were expressed as the mean $\pm \mathrm{SD}(\mathrm{n}=5) .{ }^{*} P<0.05$, vs untreated-BMSCs, ${ }^{\#} P<0.05$, vs $\mathrm{H}_{2} \mathrm{O}_{2}$ treated-BMSCs.

\section{Result}

\subsection{Ad-GDNF decreased $\mathrm{H}_{2} \mathrm{O}_{2}$-induced cytotoxicity}

As shown in Figure 1, MTT results showed the viability of BMSCs exposed to $\mathrm{H}_{2} \mathrm{O}_{2}$ was significantly decreased compared to the BMSCs without any treatment $(\mathrm{P}<0.05)$. However, when exposed to $\mathrm{H}_{2} \mathrm{O}_{2}$, the viability of GDNF/BMSCs was significantly increased compared to BMSCs $(\mathrm{P}<$ 0.05). The results indicated that GDNF suppressed the BMSCs' death induced by $\mathrm{H}_{2} \mathrm{O}_{2}$. Consistent with the results of MTT, the treatment of $\mathrm{H}_{2} \mathrm{O}_{2}$ significantly increased the LDH release in medium, but $\mathrm{LDH}$ release in GDNF/BMSCs was lower than that in BMSCs $(\mathrm{P}<0.05)$, suggesting that adenoviral-mediated GDNF decrease $\mathrm{H}_{2} \mathrm{O}_{2}$-induced cytotoxicity.

\subsection{Ad-GDNF inhibited apoptosis of BMSCs induced by $\mathrm{H}_{2} \mathrm{O}_{2}$}

To determine whether Ad-GDNF inhibits apoptosis of BMSCs induced by $\mathrm{H}_{2} \mathrm{O}_{2}$, the apoptosis of BMSCs in the presence or absence of Ad-GDNF was assessed by DAPI and TUNEL staining. As shown in Figure 2, DAPI staining revealed that many condensed nuclei or apoptotic bodies were observed in BMSCs after exposed to $\mathrm{H}_{2} \mathrm{O}_{2}$, however, administration of Ad-GDNF decreased significantly apoptosis of BMSCs compared with treatment with $\mathrm{H}_{2} \mathrm{O}_{2}$ alone. TUNEL staining was used to quantify the number of apoptotic cells induced by $\mathrm{H}_{2} \mathrm{O}_{2}$. BMSCs treated with Ad-GDNF showed significant decreases in the ratio of TUNEL positive cells compared to vehicle-treated cells $(68.11 \% \pm 6.63 \%$ vs. $35.89 \% \pm 3.04 \%, \mathrm{P}<0.05)$. 


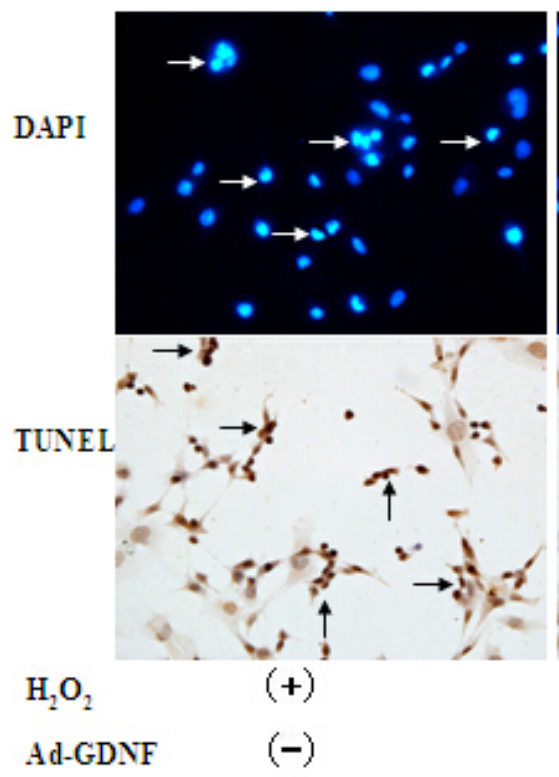

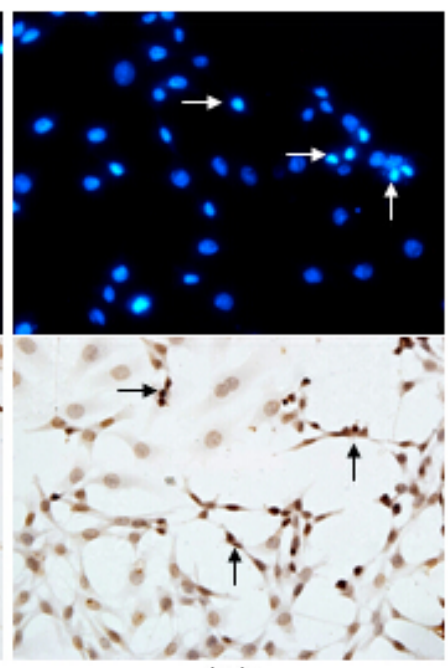

(+)

(+)

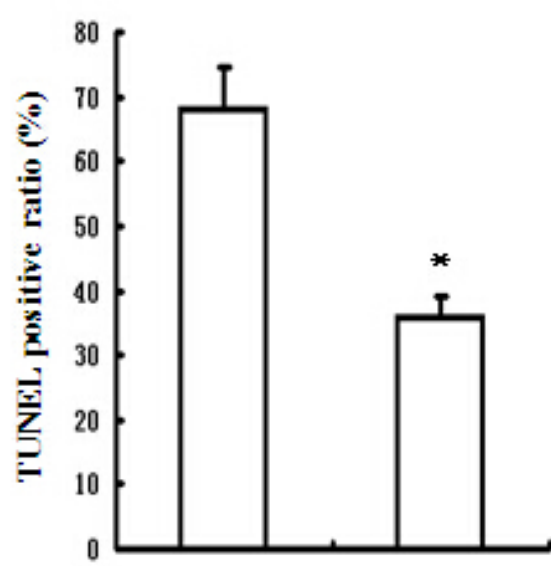

$(+)$

(+)

$(-)$

(+)

Fig. 2. Inhibitory effect of Ad-GDNF on $\mathrm{H}_{2} \mathrm{O}_{2}$-induced apoptosis in BMSCs. Data were expressed as the mean $\pm \mathrm{SD}(\mathrm{n}=5)$. ${ }^{*} \mathrm{P}<0.05$, vs $\mathrm{H}_{2} \mathrm{O}_{2}$-treated BMSCs.

Table 1

GDNF expression in BMSCs and GDNF/BMSCs (mean \pm SD)

\begin{tabular}{lllll}
\hline \multirow{2}{*}{ Groups } & \multirow{2}{*}{ BMSCs } & \multicolumn{2}{l}{ GDNF/BMSCs } & \\
\cline { 2 - 4 } & & passage 1 & passage 2 & passage 3 \\
\hline GDNF protein $(\mathrm{pg} / \mathrm{mL})$ & $38.33 \pm 2.53$ & $87.38 \pm 6.88^{*}$ & $83.48 \pm 9.88^{*}$ & $85.10 \pm 8.53^{*}$ \\
\hline \multicolumn{5}{c}{ Note: ${ }^{*} P<0.05, v s$ BMSCs. }
\end{tabular}

Table 2

Effects of Ad-GDNF on the expressions of IL- 6 and TNF- $\alpha$ and Bcl-2/Bax ratio in BMSCs under $\mathrm{H}_{2} \mathrm{O}_{2}$-induced oxidative stress (mean $\pm \mathrm{SD}, \mathrm{n}=5$ )

\begin{tabular}{lll}
\hline & $\mathrm{H}_{2} \mathrm{O}_{2}$ - treated BMSCs & $\mathrm{H}_{2} \mathrm{O}_{2}$ - treated GDNF/BMSCs \\
\hline IL-6 protein $(\mathrm{pg} / \mathrm{mL})$ & $68.11 \pm 6.63$ & $35.89 \pm 3.04^{*}$ \\
TNF- $\alpha$ protein $(\mathrm{pg} / \mathrm{mL})$ & $782.72 \pm 69.65$ & $467.34 \pm 39.92^{*}$ \\
Bcl-2/Bax ratio $(\%)$ & $7.18 \pm 1.44$ & $16.03 \pm 1.24^{*}$ \\
\hline
\end{tabular}

\subsection{Ad-GDNF increased GDNF expression in BMSCs}

To ascertain whether Ad-GDNF increases GDNF expression in BMSCs, ELISA assays were performed to test the content of GDNF protein in GDNF/BMSCs and BMSCs. As shown in Table 1, GDNF/BMSCs had higher GDNF protein expression than BMSCs $(\mathrm{P}<0.05)$, however, the contents in passage 1, 2, and 3 of GDNF/BMSCs had no significantly difference $(\mathrm{P}>0.05)$. 


\subsection{Ad-GDNF restrained secretion of IL-6 and TNF- $\alpha$ from BMSCs induced by $\mathrm{H}_{2} \mathrm{O}_{2}$}

To investigate the potential mechanism by which Ad-GDNF inhibits $\mathrm{H}_{2} \mathrm{O}_{2}$-induced apoptosis in BMSCs, the secretion of pro-inflammatory cytokines, IL- 6 and TNF- $\alpha$ from BMSCs when exposed to $\mathrm{H}_{2} \mathrm{O}_{2}$ was detected. ELISA analysis revealed under $\mathrm{H}_{2} \mathrm{O}_{2}$-induced oxidative stress, GDNF/BMSCs lowered the expressions of IL-6 and TNF- $\alpha$ with respect to BMSCs $(\mathrm{P}<0.05)$, suggesting that treatment of Ad-GDNF significantly inhibite the $\mathrm{H}_{2} \mathrm{O}_{2}$-induced secretion of IL- 6 and TNF- $\alpha$ from BMSCs, as shown in Table 2.

\subsection{Ad-GDNF up-regulated Bcl-2/Bax ratio in BMSCs under oxidative stress}

To investigate whether apoptosis was involved in protective effect of Ad-GDNF on BMSCs exposed to $\mathrm{H}_{2} \mathrm{O}_{2}$, the expressions of anti-apoptotic protein, Bcl-2 and pro-apoptotic protein, Bax were detected. It has been believed that the $\mathrm{Bcl}-2 / \mathrm{Bax}$ ratio may be more crucial than either promoter alone to prevent apoptosis [9]. Therefore, Bcl-2/Bax ratio in cell pellets was estimated by ELISA analysis. The results showed under oxidative stress, the Bcl-2/Bax ratio in GDNF/BMSCs increased significantly compared to BMSCs $(\mathrm{P}<0.05)$, suggesting that Ad-GDNF up-regulate $\mathrm{Bcl}-2 / \mathrm{Bax}$ ratio in BMSCs after exposure to $\mathrm{H}_{2} \mathrm{O}_{2}$, as shown in Table 2 .

\section{Discussion}

Oxidative stress is one of major mechanisms underlying the pathogenesis of neurodegenerative diseases [10,11] and chronic inflammatory diseases [12]. Although previous studies indicate transplantation of GDNF/BMSCs contributed to relief of functional deficits in rats following stroke [6], the mechanisms are unknown. The oxidative stress contributes to pathogenesis of CNS diseases [13]. Therefore, the effect of GDNF/BMSCs on oxidative stress such as $\mathrm{H}_{2} \mathrm{O}_{2}$ in vitro was investigated.

In present, MTT and LDH analyses showed that viability of BMSCs was significantly decreased after exposure to $\mathrm{H}_{2} \mathrm{O}_{2}$ and Ad-GDNF significantly reversed this effect. These results suggest that Ad-GDNF protects BMSCs against oxidative stress, which are consistent with the previous reports [14-16]. It has been reported that GDNF exerts neuroprotection against $\mathrm{H}_{2} \mathrm{O}_{2}$ by increasing glutathione peroxidase-1 [14,15] and decreasing heme oxygenase-1 [16]. In addition, apoptosis was detected by TUNEL and DAPI staining. The results showed that Ad-GDNF significantly decreased the apoptosis of BMSCs when exposed to $\mathrm{H}_{2} \mathrm{O}_{2}$. The results confirmed that death of BMSCs induced by $\mathrm{H}_{2} \mathrm{O}_{2}$ mainly results from apoptosis, and GDNF partly blocks this apoptosis. GDNF has been found to have powerful anti-apoptotic effects at various insults, such as radiation, spinal cord injury and 6-hydroxydopamine [17,18]. However, the exact mechanisms need to explore.

When they were exposed to $\mathrm{H}_{2} \mathrm{O}_{2}$, BMSCs were injured and maybe release the proinflammatory cytokines, which in turn damage the BMSCs and induce the apoptosis of BMSCs. IL- 6 and TNF- $\alpha$, potent proinflammatory cytokines, play a critical role in initiating and sustaining the inflammatory response [19]. Meantime, IL-6 and TNF- $\alpha$ are also pro-apoptotic cytokines because they promote neuronal apoptosis induced by hypoxia-ischemia [20]. In present, the levels of TNF- $\alpha$ and IL-6 from the supernatants in cultures were detected after BMSCs were exposed to $\mathrm{H}_{2} \mathrm{O}_{2}$. The levels of TNF- $\alpha$ and IL-6 in GDNF/BMSCs cultures were significantly reduced compared to BMSCs cultures. Given that GDNF content in the supernatants from GDNF/BMSCs cultures were significantly increased 
compared to BMSCs cultures, it can be supposed that GDNF secreted from BMSCs may inhibit TNF- $\alpha$ and IL-6 secretion from BMSCs, which may induce apoptosis of BMSCs exposed to $\mathrm{H}_{2} \mathrm{O}_{2}$.

Moreover, previous reports have shown that pretreatment of GDNF prevented the apoptotic effects by the up-regulation of the anti-apoptotic protein, Bcl-w and Bcl-2, as well as the down-regulation of the pro-apoptotic proteins, Bad and Bax [18]. The up-regulating levels of GDNF inhibit the expression of the Bax and caspase-3 in $\mathrm{H}_{2} \mathrm{O}_{2}$-treated astrocytes [21]. These results indicate that GDNF protection against apoptosis is related to modulating the expression of apoptotic protein. Therefore, the $\mathrm{Bcl}-2 / \mathrm{Bax}$ ratio was investigated under $\mathrm{H}_{2} \mathrm{O}_{2}$ oxidative conditions. The results showed that $\mathrm{Bcl}-2 / \mathrm{Bax}$ ratio in GDNF/BMSCs cultures was higher with respect to BMSCs cultures, which suggested that GDNF prevent apoptosis by modulating Bcl-2/Bax ratio. The results in vitro are consistent with previous results in which transplantation of GDNF/BMSCs has been shown to lessen number of apoptotic cells compared with transplantation of BMSCs alone after cerebral hemorrhage [6].

In conclusion, Ad-GDNF inhibits apoptosis of BMSCs induced by $\mathrm{H}_{2} \mathrm{O}_{2}$, and the mechanisms may be related to down-regulating the expression of IL- 6 and TNF- $\alpha$ and up-regulating the ratio of Bcl-2/Bax in BMSCs.

\section{Acknowledgement}

This study was supported by the National Natural Science Foundation of China (No. 81371213).

\section{References}

[1] A.H. Schapira and P. Jenner, Etiology and pathogenesis of Parkinson's disease, Mov. Disord. 26 (2011), $1049-1055$.

[2] L.F. Lin, D.H. Doherty, J.D. Lile, S. Bektesh and F. Collins, GDNF: a glial cell line-derived neurotrophic factor for midbrain dopaminergic neurons, Science 260 (1993), 1130-1132.

[3] C.E. Henderson, H.S. Phillips, R.A. Pollock, A.M. Davies, C. Lemeulle, M. Armanini, L. Simmons, B. Moffet, R.A. Vandlen and L.C. Simpson, GDNF: a potent survival factor for motoneurons present in peripheral nerve and muscle, Science 266 (1994), 1062-1064.

[4] A. Bakshi, S. Shimizu, C.A. Keck, S. Cho, D.G. LeBold, D. Morales, E. Arenas, E.Y. Snyder, D.J. Watson and T.K. McIntosh, Neural progenitor cells engineered to secrete GDNF show enhanced survival, neuronal differentiation and improve cognitive function following traumatic brain injury, Eur. J. Neurosci. 23 (2006), 2119-2134.

[5] A.K. Chou, M,C. Yang, H.P. Tsai, C.Y. Chai, M.H. Tai, A.L. Kwan and Y.R. Hong, Adenoviral-mediated glial cell line-derived neurotrophic factor gene transfer has a protective effect on sciatic nerve following constriction-induced spinal cord injury, PLoS One 9 (2014), e92264-1-e92264-12.

[6] C.X. Yang, L. Zhou, X.Q. Gao, B. Chen, J. Tu, H. Sun, X. Liu, J. He, J, Liu and Q.L. Yuan, Neuroprotective effects of bone marrow stem cells overexpressing glial cell line-derived neurotrophic factor on rats with intracerebral hemorrhage and neurons exposed to hypoxia/reoxygenation, Neurosurgery 68 (2011), 691-704.

[7] B. Chen, X.Q. Gao, C.X. Yang, S.K. Tan, Z.L. Sun, N.H. Yan, Y.G. Pang, M. Yuan, G.J. Chen, G.T. Xu, K. Zhang and Q.L. Yuan, Neuroprotective effect of grafting GDNF gene-modified neural stem cells on cerebral ischemia in rats, Brain Res. 1284 (2009), 1-11.

[8] J. Du, X.Q. Gao, L. Deng, N.B. Chang, H.L. Xiong and Y. Zheng, Transfection of the glial cell line-derived neurotrophic factor gene promotes neuronal differentiation, Neural Regeneration Res. 9 (2014), 33-40.

[9] P.G. Del, A. Venditti, P.M. Del, L. Maurillo, F. Buccisano, A. Tamburini, M.C. Cox, A. Franchi, A. Bruno, C. Mazzone, P. Panetta, G. Suppo, M. Masi and S. Amadori, Amount of spontaneous apoptosis detected by Bax/Bcl-2 ratio predicts outcome in acute myeloid leukemia (AML), Blood 101 (2003), 2125-2131.

[10] D.A. Patten, M. Germain, M.A. Kelly and R.S. Slack, Reactive oxygen species: stuck in the middle of neurodegeneration, J. Alzheimers Dis. 20 (2010), S357-S367. 
[11] L. Nanetti, F. Raffaelli, A. Vignini, C. Perozzi, M. Silvestrini, M. Bartolini, L. Provinciali and L. Mazzanti, Oxidative stress in ischaemic stroke, Eur. J. Clin. Invest. 41 (2011), 1318-1322.

[12] G.X. Liu, Y.X. Yang, J. Yan, T. Zhang, Y.P. Zou, X.L. Huang and H.T. Gan, Glial-derived neurotrophic factor reduces inflammation and improves delayed colonic transit in rat models of dextran sulfate sodium-induced colitis, Int. Immunopharmacol. 19 (2014), 145-152.

[13] F.W. Wang, Z. Wang, Y.M. Zhang, Z.X. Du, X.L. Zhang, Q. Liu, Y.J. Guo, X.G. Li and A.J. Hao, Protective effect of melatonin on bone marrow mesenchymal stem cells against hydrogen peroxide-induced apoptosis in vitro, J. Cell Biochem. 114 (2013), 2346-2355.

[14] I.G. Onyango, J.B. Tuttle and J.P. Bennett, Brain-derived growth factor and glial cell line-derived growth factor use distinct intracellular signaling pathways to protect $\mathrm{PD}$ cybrids from $\mathrm{H}_{2} \mathrm{O}_{2}$-induced neuronal death, Neurobiol. Dis. 20 (2005), 141-154.

[15] E. Gharib, M. Gardaneh and S. Shojaei, Upregulation of glutathione peroxidase-1 expression and activity by glial cell line-derived neurotrophic factor promotes high-level protection of PC12 cells against 6-hydroxydopamine and hydrogen peroxide toxicities, Rejuvenation Res. 16 (2013), 185-199.

[16] A. Saavedra, G. Baltazar, C.M. Carvalho and E.P. Duarte, GDNF modulates HO-1 expression in substantia nigra postnatal cell cultures, Free Radic. Biol. Med. 39 (2005), 1611-1619.

[17] G. Liu, X. Wang, G. Shao and Q. Liu, Genetically modified Schwann cells producing glial cell line-derived neurotrophic factor inhibit neuronal apoptosis in rat spinal cord injury, Mol. Med. Rep. 9 (2014), 1305-1312.

[18] J.P. Cao, H.Y. Niu, H.J. Wang, X.G. Huang and D.S. Gao, NF-кB p65/p52 plays a role in GDNF up-regulating Bcl-2 and Bcl-w expression in 6-OHDA-induced apoptosis of MN9D cell, Int. J. Neurosci. 123 (2013), 705-710.

[19] T. Debnath, S.R. Park, H. Kim da, J.E. Jo and B.O. Lim, Anti-oxidant and anti-inflammatory activities of inonotus obliquus and germinated brown rice extracts, Molecules 18 (2013), 9293-9304.

[20] S.J. Li, W. Liu, J.L. Wang, Y. Zhang, D.J. Zhao, T.J. Wang and Y.Y. Li, The role of TNF- $\alpha$, IL-6, IL-10, and GDNF in neuronal apoptosis in neonatal rat with hypoxic-ischemic encephalopathy, Eur. Rev. Med. Pharmacol. Sci. 18 (2014), 905-909.

[21] S, Kim and J. Kwon, [6]-shogaol attenuates neuronal apoptosis in hydrogen peroxide-treated astrocytes through the up-regulation of neurotrophic factors, Phytother. Res. 27 (2013), 1795-1799. 